\title{
Effect of Glucans on the Antitumor Activity of Grifolan
}

\author{
NaOhito Ohno, ${ }^{a}$ Mami Hayashi, ${ }^{a}$ Kazuyoshi Iino, ${ }^{a}$ Iwao Suzuki, ${ }^{a}$ \\ Shozo OIKaWa, ${ }^{b}$ Kichiro Sato, ${ }^{b}$ YoshiYUKi SUzUKI, ${ }^{b}$ \\ and TOSHIRO YADOMAE*,a
}

Tokyo College of Pharmacy, ${ }^{a}$ Horinouchi, Hachioji, Tokyo 192-03, Japan and Nippon Beet Sugar Mfg. Co., Ltd., ${ }^{b}$ Kyobashi, Chuo-ku, Tokyo 104, Japan

(Received October 22, 1985)

\begin{abstract}
The effects of antitumor-active [ $\beta$-1,3-glucan (grifolan)] and inactive [ $\beta$-1,6(islandican), $\alpha-1,4-$ (starch)]glucans in mice were compared. $\beta$-1,6-Glucan enhanced the antitumor activity of grifolan, whereas $\alpha$-1,4-glucan showed a suppressive effect on the antitumor activity of grifolan. When these glucans were injected i.p. into normal mice, the glucans disappeared gradually. The rate of disappearance was in the order $\alpha-1,4>\beta-1,6>\beta-1,3$. Grifolan induced peritoneal exudate cells, but $\beta-1,6$ and $\alpha-1,4$-glucans did not. Only $\alpha-1,4$-glucan was degradated by the lysate of the peritoneal exudate cells. These results indicate that the antitumor activity of $\beta$-1,3-glucan can be positively or negatively modulated by other glucans, and that glucans showing different antitumor effects have a variety of actions and fates in mice.
\end{abstract}

Keywords__antitumor activity; immunomodulator; $\beta$-1,3-glucan; grifolan; glucan

\section{Introduction}

Recently, Mizuno et al. classified the immunomodulators into subgroups in relation to the appearance of a serum protein or polymorphonuclear leukocyte (PMN) response: ${ }^{1)}$ Combinations of drugs in different group might be useful for cancer therapy. Traditional herbal medicine in China and Japan is based on such a combinational approach. Interestingly, extracts of mushrooms have been used for cancer therapy in Sino-Japanese herbal medicine. ${ }^{2)}$

We have studied the structure and antitumor activity of glucans of Grifola frondosa. ${ }^{3)}$ Based on characterizations of the hot water, cold alkali and hot alkali extracts of $G$. frondosa, this fungus contains both neutral $(\alpha-1,4, \beta-1,3)$ and acidic $(\alpha-1,3, \beta-1,6)$ glucans. ${ }^{3 c)}$ The activity of the neutral fraction was owing to the $\beta$-1,3-glucan, called "grifolan." $3 d$ ) In earlier work, crude extract of $G$. frondosa (GFE) was found to show antitumor effects via p.o. and i.p. administrations. ${ }^{3 e}$ ) Further, we have studied the antitumor effect of the polysaccharide fraction GF-1, ${ }^{3 f)}$ which is a crude polysaccharide fraction composed mainly of $\alpha-1,4, \beta-1,6$ and $\beta-1,3$ linkages. GF-1 showed antitumor effect via i.p., i.v., or i.t. administrations. It also induced resistance against Sarcoma 180 in ICR mice which had completely cured of the tumor by GF-1 treatment. The administration of GF-1 to mice increased the weight or cell number of the spleen and peritoneal cavity, and enhanced the antigen-specific antibody response and carbon clearance activity. However, GF-1 showed no antitumor activity via p.o. administration, and some of these effects were different from those of purified glucans reported elsewhere.

Based on these findings, we have suggested that the mode of antitumor activity and the effects on the immune system of the antitumor glucan may be modulated by several other materials. Antitumor activity via p.o. administration or the different mode of action from other glucans might well be consistent with involvement of several materials. We wished to 
know what kind of effects were shown by the antitumor inactive polysaccharides and by combinations of antitumor active and inactive polysaccharides. This paper is concerned with the effects of antitumor active (grifolan) and inactive $(\alpha-1,4$, and $\beta-1,6)$ glucans in mice.

\section{Materials and Methods}

Determination of Number of Peritoneal Exudate Cells, and Contents of Hydrolytic Enzymes and Remaining Polysaccharides_-At an appropriate time after treatment, mice were sacrificed by exposure to ether. Peritoneal exudate cells (PEC) were recovered from each mouse with $10 \mathrm{ml}$ of saline. The number of PEC was determined by the trypan blue dye exclusion test. After centrifugation, each supernatant was used for measuring remaining polysaccharides and the packed PEC were lysed by adding $0.1 \%$ Triton X-100 solution. Enzyme activity was measured by using the Triton lysates. The remaining polysaccharide was estimated from the carbohydrate content measured by the phenol-sulfuric acid method.

Determination of Molecular Weight under Physiological Conditions_-Each glucan was dissolved in saline and the molecular weight $M_{\mathrm{r}}$ was determined by using a column of Toyopearl HW-65S. The elution profiles were monitored with a refractive index detector.

Other Methods-Islandican was prepared from the culture filtrate of Penicillium islandicum. ${ }^{4)}$ Other materials were from commercial sources. Mice were purchased from the Shizuoka Agricultural Cooperative Association for Laboratory Animals, Hamamatsu. All mice used in experiments were 2 to 3 months old, male ICR strain. Other methods were as described previously. ${ }^{3)}$

\section{Results}

\section{Effects of Glucans on the Antitumor Activity of Grifolan}

Recently, we showed that the mode of antitumor activity of the polysaccharide fraction, GF-1, was different from those of other antitumor substances. $\left.{ }^{3 f}\right)$ It was considered that $\beta$ 1,3-glucan and other substances ( $\beta$-1,6 and $\alpha$-1,4-glucans) might all be important for the antitumor activity of GF-1. Thus, we examined the effects of $\beta-1,6$ and $\alpha-1,4$-glucans on the mode of action of antitumor activity of grifolan. As the glucan sources, islandican $(\beta-1,6-$ glucan obtained from Penicillium islandicum) and starch $(\alpha-1,4$-glucan) were used. Table I showed the antitumor effects of these glucans on Sarcoma 180 in mice. Only $\beta$-1,3-glúcan showed antitumor activity. As the next step, combinations of grifolan and islandican or starch were examined. The doses of these glucans were as follows: grifolan, 4, 7.5, $20 \mu \mathrm{g} / \mathrm{mouse}$; other glucans, $2000 \mu \mathrm{g}$. This is similar to the ratio in GF-1. As shown in Table II and Fig. 1, cooperativity was detected at the doses of 4 and $7.5 \mu \mathrm{g} / \mathrm{mouse} / \mathrm{d}$. Isolandican augmented the activity of grifolan and starch suppressed it $(4 \mu \mathrm{g}, p<0.01 ; 7.5 \mu \mathrm{g}, p<0.05)$. These results suggest that the antitumor activity of $\beta$-1,3-glucan is modulated by other glucans.

\section{Effect of Glucans on the Number of Cells in the Peritoneal Cavity}

For clarifying the effects of glucans on the peritoneal cavity, the cell number on day 1 or 4 after treatment with glucans was examined (Table III). In the cases of the antitumor-active

TABLE I. Effects of Glucans ${ }^{a)}$ on the Growth of Sarcoma 180 in ICR Mice

\begin{tabular}{lcccc}
\hline Glucan & $\begin{array}{c}\text { Dose } \\
(\mu \mathrm{g} / \text { mouse } \times 5)\end{array}$ & $\begin{array}{c}\text { Tumor weight } \\
(\mathrm{g}, \text { mean } \pm \text { S.D. })\end{array}$ & $\begin{array}{c}\text { Inhibition }^{b)} \\
(\%)\end{array}$ & $\begin{array}{c}\text { Complete } \\
\text { regression }^{b)}\end{array}$ \\
\hline Grifolan & 100 & $<0.1^{d)}$ & $>99$ & $3 / 9$ \\
Islandican & 4000 & $11.9 \pm 7.3$ & 0 & $0 / 6$ \\
Starch & 4000 & $10.6 \pm 5.2$ & 12 & $0 / 6$ \\
Nil & & $12.0 \pm 4.3$ & - & $0 / 16$ \\
\hline
\end{tabular}

a) Sarcoma 180 tumor cells $\left(5 \times 10^{6}\right)$ were inoculated subcutaneously. Each sample was administered 5 times on day $1,3,5,7$ and 9 as a saline solution by intraperitoneal injection. b) "Inhibition" and "complete regression" were determined at day 35 after the tumor inoculation. c) The significance was evaluated by means of Student's $t$-test. $d$ ) $p<0.001$ 
TABLE II. Modulation of Antitumor Effect of Grifolan by Glucans ${ }^{a)}$

\begin{tabular}{lcccc}
\hline \hline Glucan & $\begin{array}{c}\text { Dose } \\
(\mu \mathrm{g} / \text { mouse } \times 10)\end{array}$ & $\begin{array}{c}\text { Tumor weight } \\
(\mathrm{g}, \text { mean } \pm \text { S.D. })\end{array}$ & $\begin{array}{c}\text { Inhibition } \\
(\%)\end{array}$ & $\begin{array}{c}\text { Complete } \\
\text { regression }^{b)}\end{array}$ \\
\hline Grifolan & 4 & $1.8 \pm 1.5^{e)}$ & 74 & $1 / 9$ \\
& 7.5 & $0.5 \pm 0.8^{e)}$ & 93 & $3 / 9$ \\
& 20 & $0.4 \pm 0.6^{e)}$ & 93 & $1 / 10$ \\
Grifolan & $4 \quad(2000)$ & $2.8 \pm 1.8^{d, f)}$ & 58 & $1 / 10$ \\
(Starch) & $7.5(2000)$ & $1.0 \pm 1.0^{e, g)}$ & 85 & $0 / 10$ \\
& $20 \quad(2000)$ & $0.6 \pm 1.0^{e)}$ & 91 & $2 / 10$ \\
Grifolan & $4(2000)$ & $0.9 \pm 0.9^{e, f)}$ & 86 & $3 / 10$ \\
(Islandican) & $7.5(2000)$ & $0.1 \pm 0.2^{e, g)}$ & 98 & $3 / 10$ \\
Nil & $20(2000)$ & $0.4 \pm 0.8^{e)}$ & 95 & $2 / 9$ \\
& & $6.6 \pm 3.7$ & 0 & $0 / 19$ \\
\hline
\end{tabular}

a) Sarcoma 180 tumor cells $\left(5 \times 10^{6}\right)$ were inoculated subcutaneously (day 0 ). Each sample was administered for 10 consecutive days as a saline solution by intraperitoneal injection. b) "Inhibition" and "complete regression" were determined at day 35 after tumor inoculation. c) The significance was evaluated by means of Student's $t$-test against glucan-untreated groups $(d) p<0.01 ; e) p<0.001$ ). The differences between both groups were statistically significant. $(f) p<0.01 ; g) p<0.05$ ).

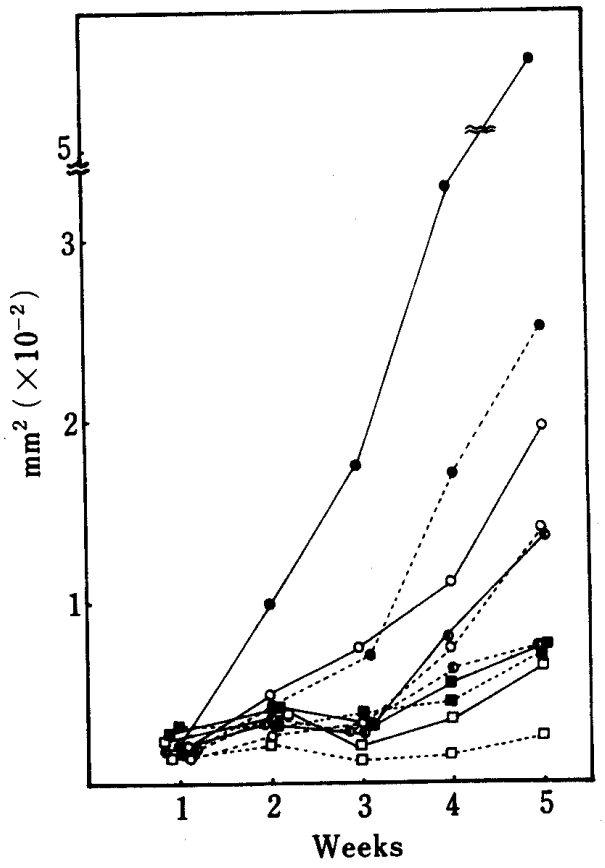

Fig. 1. Kinetics of Sarcoma 180 Growth in ICR Mice Treated with Grifolan Together with Glucans

Experimental details were the same as given in Table II. Tumor size is shown as $\mathrm{mm}^{2}$. - control; $-\mathrm{O}-$, grifolan $(4 \mu \mathrm{g})$; ---@---, grifolan $(4 \mu \mathrm{g})+$ starch; $-\mathrm{O}-$, grifolan $(4 \mu \mathrm{g})+$ islandican; - - - grifolan $(7.5 \mu \mathrm{g}) ;--\mathrm{O}_{-\cdots}$, grifolan $(7.5 \mu \mathrm{g})+$ starch; --- $\square---$, grifolan $(7.5 \mu \mathrm{g})+$ islandican; --- $\square---$, grifolan $(20 \mu \mathrm{g}) ;-\cdots-\cdots--$, grifolan $(20 \mu \mathrm{g})+$ starch; $\rightarrow-$, grifolan $(20 \mu \mathrm{g})+$ islandican

glucans (GF-1 and grifolan), the number of PEC was increased. On the other hand, the other glucans showed little or no activity for increasing PEC. The optimum doses for antitumor activity of GF-1 and grifolan have been found to be $2000 \times 10$ and $20 \times 10$, respectively. The increment of PEC at day 4 is probably related to the antitumor activity of these polysaccharides.

\section{Comparison of the Disappearance Rate of Glucans from the Peritoneal Cavity}

To clarify the fate of glucans in the peritoneal cavity, clearance time of glucans from the peritoneal cavity were compared. For determining the remaining glucan content, at an appropriate time, the peritoneal cavity was washed with saline and the peritoneal exudate cells were removed by centrifugation. The glucan content in the supernatant was estimated by the phenol-sulfuric acid method. In the non-treated group, phenol- $\mathrm{H}_{2} \mathrm{SO}_{4}$-positive materials 
TABLE III. Total Numbers of Peritoneal Exudate Cells after Treatment with Various Glucans ${ }^{a}$ )

\begin{tabular}{lrrr}
\hline \hline Glucan & $\begin{array}{c}\text { Dose } \\
(\mu \mathrm{g})\end{array}$ & $\begin{array}{c}\text { Day } 1 \\
\left(\times 10^{-6} / \text { mouse }\right)\end{array}$ & $\begin{array}{c}\text { Day } 4 \\
\left(\times 10^{-6} / \text { mouse }\right)\end{array}$ \\
\hline GF-1 & 80 & $6.0 \pm 1.6$ & $5.6 \pm 2.2$ \\
& 400 & $6.4 \pm 2.0$ & $7.6 \pm 2.2$ \\
Starch & 2000 & $15.8 \pm 2.8$ & $13.6 \pm 3.6$ \\
& 80 & $6.0 \pm 1.0$ & $7.0 \pm 1.8$ \\
Dextran & 400 & $5.0 \pm 0.8$ & $6.0 \pm 1.8$ \\
& 2000 & $2.4 \pm 1.0$ & $7.6 \pm 3.6$ \\
Grifolan & 80 & $3.2 \pm 1.4$ & $5.6 \pm 2.4$ \\
& 400 & $9.2 \pm 3.6$ & $6.0 \pm 1.2$ \\
& 2000 & $6.6 \pm 4.4$ & $5.0 \pm 2.2$ \\
Islandican & 80 & $5.2 \pm 2.8$ & $18.8 \pm 9.4$ \\
& 400 & $12.8 \pm 0.4$ & $12.6 \pm 3.4$ \\
& 2000 & $31.8 \pm 5.0$ & $16.2 \pm 6.4$ \\
Nil & 80 & $6.2 \pm 2.0$ & $7.0 \pm 1.4$ \\
& 400 & $3.6 \pm 1.4$ & $8.2 \pm 2.2$ \\
& 2000 & $5.2 \pm 1.4$ & $5.4 \pm 2.8$ \\
& & $6.0 \pm 1.4$ & $5.8 \pm 1.6$ \\
\hline
\end{tabular}

a) In each group, 3 mice were used, and PEC was determined by the trypan blue dye exclusion test. Glucan (80, 400, or $2000 \mu \mathrm{g} / 0.2 \mathrm{ml}$ saline) was injected i.p. at day 0 . After 1 (day 1) or 4 (day 4) d, mice were killed by exposure to ether. Peritoneal exudate cells were recovered by washing the peritoneal cavity with saline.

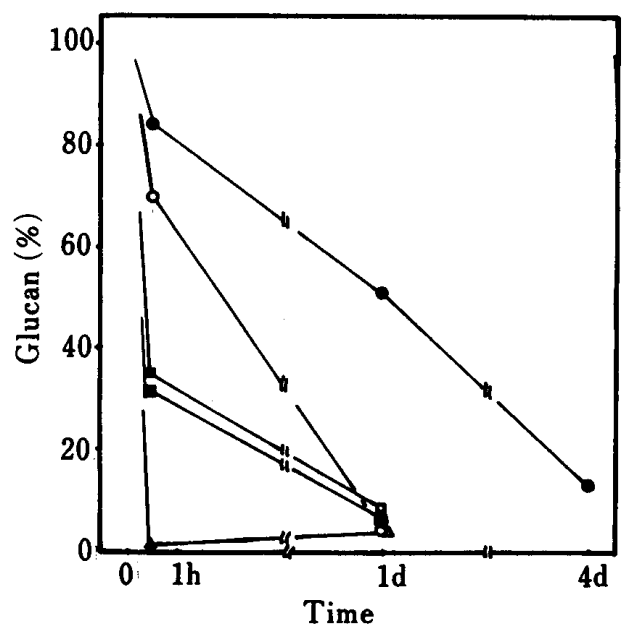

Fig. 2. Comparison of the Disappearance Rates of Glucans from the Peritoneal Cavity

Glucan remaining in the peritoneal cavity was determined by measurement of total carbohydrate. -O-, grifolan; - O-, islandican; - $\square-$, dextran; $-\square-$ GF-1; $-\triangle-$, starch.

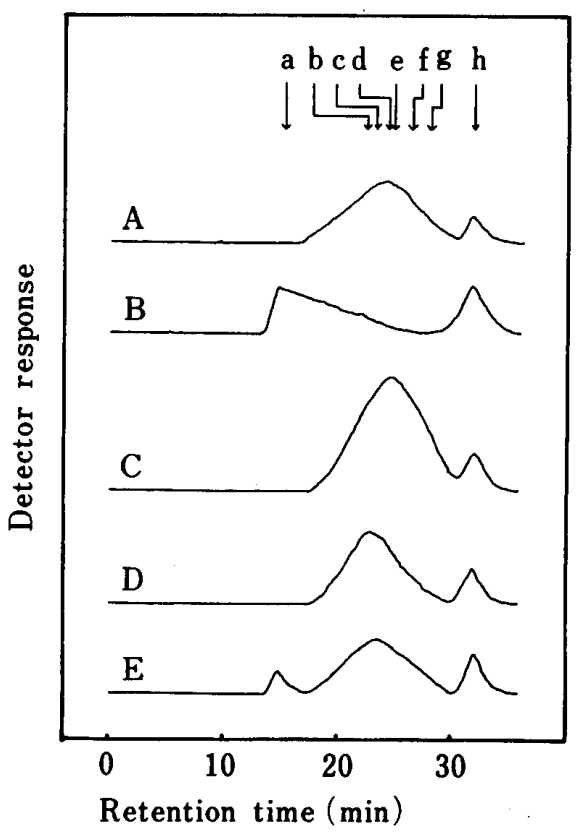

Fig. 3. Elution Profiles of Various Glucans from a Column of Toyopearl HW-65S

Each glucan dissolved in saline was applied to a column of Toyopearl HW- $65 \mathrm{~S}(1.6 \times 45 \mathrm{~cm})$ equilibrated with saline. The flow rate was $0.5 \mathrm{ml} / \mathrm{min}$ and the elution profiles were monitored with a refractive index detector. A, starch; B, grifolan; C, islandican; D, GF-1; E, dextran T-2000, a, Vo; b, dextran T-2000; c, T-250; d, T-100; e, T-70; f, T-40; g, T-10; h, Vt. The peak at $\mathrm{Vt}$ is due to the presence of salt. 


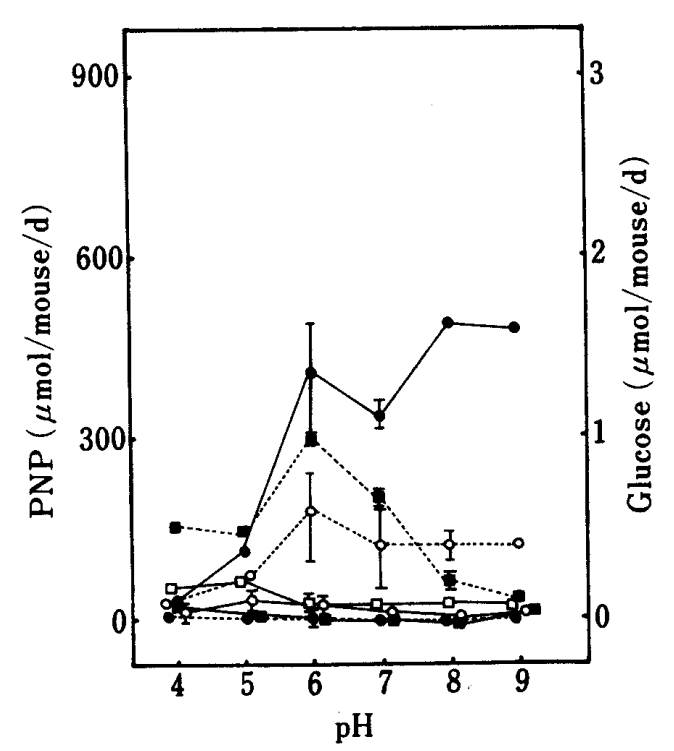

Fig. 4. Hydrolytic Enzyme Activity of Peritoneal Exudate Cells Obtained at Day 1 after Thioglycolate Broth Treatment

Hydrolytic enzyme activity of the Triton lysates was measured at $37^{\circ} \mathrm{C}$ at various $\mathrm{pH}$ values. Substrates were --- -.-, $p$-nitrophenyl- $\alpha$-glucoside; - $\square-$, $p$-nitrophenyl- $\beta$-glucoside; - - , starch; --.O--, GF-1; - - - , dextran T-110; - O-, islandican; ------, grifolan. Hydrolytic enzyme activities toward polysaccharides were assessed by reducing power measurement.

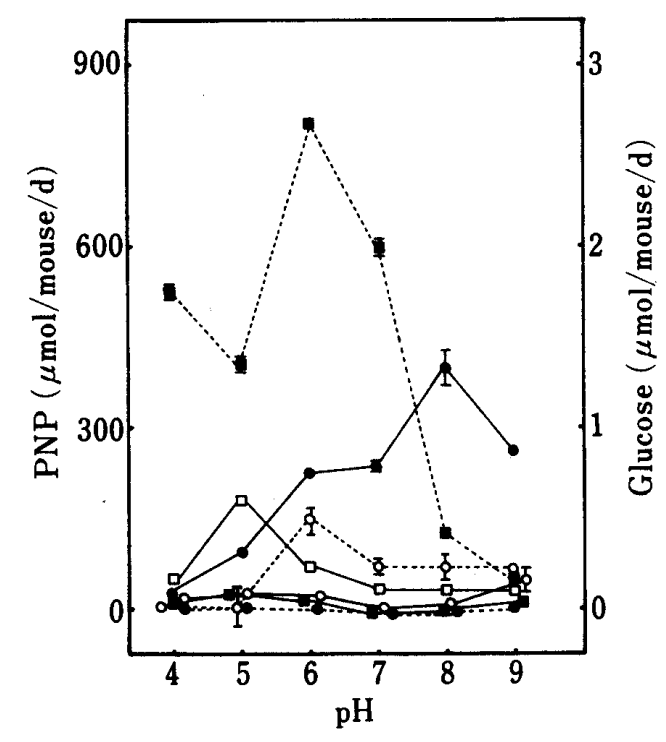

Fig. 5. Hydrolytic Enzyme Activity of Peritoneal Exudate Cells Obtained at Day 4 after Thioglycolate Broth Treatment

Experimental conditions and symbols were the same as in Fig. 4.

were contained in the peritoneal cavity at a level of about $500 \mu \mathrm{g} / \mathrm{mouse}$. The remaining glucan contents were estimated by subtraction. The remaining percentage of each glucan is shown in Fig. 2. The loss of $\alpha$-1,4-glucan was the fastest, while the loss of $\beta$-1,3-glucan was the slowest (ca. 50\%, day 1). All glucans except for grifolan had disappeared within $1 \mathrm{~d}$ after the injection. GF-1 contained a small amount of a $\beta$-1,3-glucan, which also disappeared within $1 \mathrm{~d}$. It is assumed that the remaining percentage is lower than the limit of the quantitative analysis, because the $\beta-1,3$-glucan content in GF-1 is quite low.

\section{Comparison of the Molecular Weights of Glucans}

To clarify why the disappearance rates of glucans were different, the molecular weights of these glucans under physiological conditions were compared. Figure 3 showed the elution profiles of several glucans from Toyopearl HW-65S buffered in saline. Arrows indicated the elution volumes of standard dextrans. The average molecular weight was the highest for grifolan and the others showed similar molecular weight distributions. Grifolan was mostly eluted near the void volume of this column. This suggests that the average molecular weight of grifolan is $>2 \times 10^{6}$. On the other hand, in the case of $8 \mathrm{M}$ urea/ $0.2 \mathrm{~N} \mathrm{NaOH}$ as the solvent, grifolan was eluted at the average $M_{\mathrm{r}}$ of $7.5 \times 10^{5} .^{3 d)}$ It appears that grifolan forms aggregates by inter-chain interactions under physiological conditions.

\section{Effect of Hydrolytic Enzymes on the Disappearance of Glucans from the Peritoneal Cavity}

To elucidate the fate of glucans in the peritoneal cavity more precisely, the glucanhydrolytic capacity of peritoneal cells was examined (Figs. 4 and 5). Triton lysate of thioglycolate medium-induced peritoneal cells (day 1 and day 4) was used as the enzyme source. $p$-Nitrophenyl derivatives were hydrolyzed easily. However, in the case of glucans, only starch could be hydrolyzed by the Triton lysate. Digestion of the polymer into small molecules would accerelate the disappearance rate. 


\section{Discussion}

The present results indicate that glucans may enhance $(\beta-1,6)$ or decrease $(\alpha-1,4)$ the antitumor activity of grifolan. So, far, the most important problem in the development of antitumor glucans has been to obtain highly purified (homogeneous) materials. However, the data described in this paper raise the possibility of combination therapy. Many fungal extracts are used in Sino-Japanese herbal medicine for cancer therapy, and this presumably corresponds to combination therapy with unknown naturally occurring substances. Chemical characterization of some of these medicines has been started in recent years.

In a previous paper, we have shown that the mode of antitumor activity and the effects on the immune system of GF-1, which is a crude polysaccharide fraction composed of $\alpha-1,4, \beta$ 1,6 , and $\beta-1,3$ linkages, are different from those of the purified glucans. ${ }^{3 c)}$ The data presented in this paper suggest that the cooperation of the three kinds of glucans is one of the reasons for the differences. Cooperativity of immunomodulators in cancer therapy has also been reported. ${ }^{1)}$ However, it is necessary to investigate many polysaccharides to generalize the above observations.

We also found that antitumor-active and other glucans have different behavior and fates in the peritoneal cavity (Table III, Figs. 2, 4 and 5). Only the antitumor-active glucan induced PEC and remained for a long time in the peritoneal cavity. It is possible that the antitumor $\beta$ 1,3-glucan forms a gel (triple helix). As shown in Fig. 3, grifolan showed the highest molecular weight among the glucans tested. The fact that the elution volume of grifolan is smaller than that of dextran T-2000 indicates that the molecular weight of grifolan in the physiological state is more than $2 \times 10^{6}$. However, the molecular weight under denaturing conditions $(8 \mathrm{M}$ urea $/ 0.2 \mathrm{~N} \mathrm{NaOH}$ ) was $7.5 \times 10^{5}$. These results suggest that interchain bonding occurs under physiological conditions. On the other hand, other glucans showed smaller molecular weights. The velocity of disappearance from the peritoneal cavity might be closely related to molecular weight under physiological conditions. Hydrolysis of starch by the lysate of peritoneal cells also supports the above assumption (Figs. 4 and 5).

Seljelid et al. reported that the chemical cross-linking of laminarin induced the appearance of $\mathrm{C} 3 \mathrm{~b}$ binding capacity. ${ }^{5)}$ Further, cross-linked dextran (Sephadex) stimulated the effector cells to in vitro cytocidal activity against tumors. ${ }^{6)}$ These findings and the data presented in this paper suggest that the molecular weight of the glucan under physiological conditions is one of the most important factors for antitumor activity. It will be necessary to compare many glucans to confirm this hypothesis.

\section{References}

1) a) O. Yoshioka, S. Abe, Y. Masuko, and D. Mizuno, Gann, 72, 471 (1981); b) K. Morikawa, Y. Kikuchi, S. Abe, M. Yamazaki, and D. Mizuno, ibid., 75, 370 (1984); K. Morikawa, R. Takeda, M. Yamazaki, and D. Mizuno, Cancer Res., 45, 1496 (1985); K. Morikawa, S. Kamegaya, M. Yamazaki, and D. Mizuno, ibid., 45, 3482 (1985).

2) G. Chihara, "Gann To Meneki Zokyo," Kodansha, Tokyo, 1980.

3) a) K. Iino, N. Ohno, I. Suzuki, T. Miyazaki, T. Yadomae, S. Oikawa, and K. Sato, Carbohydr. Res., 141, 111 (1985); b) N. Ohno, I. Suzuki, K. Sato, S. Oikawa, T. Miyazaki, and T. Yadomae, Chem. Pharm. Bull., 33, 4522 (1985); c) N. Ohno, K. Iino, I. Suzuki, S. Oikawa, K. Sato, T. Miyazaki, and T. Yadomae, ibid., 33, 1181 (1985); d) N. Ohno, K. Iino, T. Takeyama, I. Suzuki, K. Sato, S. Oikawa, T. Miyazaki, and T. Yadomae, ibid., 33, 3395 (1985); e) T. Miyazaki, T. Yadomae, I. Suzuki, M. Nishijima, S. Yui, S. Oikawa, and K. Sato, Jpn. J. Med. Mycol., 23, 261 (1982); f) I. Suzuki, T. Itani, N. Ohno, S. Oikawa, K. Sato, T. Miyazaki, and T. Yadomae, J. Pharmacobio-Dyn., 7, 492 (1984); idem, ibid., 8, 217 (1985).

4) N. Nakamura, J. Ooyama, and O. Tanabe, Nippon Nogeikagaku Kaishi, 35, 949 (1961); E. Ebert and M. H. Zzenk, Arch. Mikrobiol., 54, 276 (1966).

5) R. Seljelid, J. Bogwald, and A. Lundwall, Exp. Cell Res., 131, 121 (1981).

6) C. A. Blanckmeister and D. H. Sussdorf, J. Leu. Biol., 37, 209 (1985). 